\title{
A New Approach for Estimating Free Point in Fishing of Stuck Pipe using Artificial Neural Network
}

\author{
Aboutaleb Sasan Nejad \\ Petroleum University of Technology \\ Ahwaz, Iran
}

\author{
Khalil Shahbazi \\ Petroleum University of Technology \\ Ahwaz, Iran
}

\begin{abstract}
Stuck pipe is a common problem in drilling industry. It accounts for the major rig time losses each year in the petroleum industry. In cases where common solutions such as pulling up and pushing down, rotating, jarring, and changing flow rate don't work, then backing off is the last resort. To have a successful back off operation, estimating location of the free point is vital.

In this study, an attempt is made to estimate the free point in stuck pipe cases using the drilling data and artificial neural network approach. For this purpose, drilling data such as mud properties, pipe rotation, rate of penetration, and some other parameters are required. In this study, artificial neural networks (ANNs) model using field data from more than 40 wells was employed, and results were compared to field results. ANN model was constructed with a supervised learning algorithm and feed forward back propagation learning rule is used for training the network. The statistical error analysis results obtained by the models and acceptable values for correlation coefficient indicate that ANN model is successful in free point prediction.
\end{abstract}

\section{Keywords}

Free point, stuck pipe, artificial neural network.

\section{INTRODUCTION}

Generally, stuck pipe is described as any restriction of upward or/and downward movement of drill string or/and pipe rotation. This condition will lead to stop drilling operation as no more drilling is achieved. Pipe sticking could occur due to many causes. The most common two types of stuck pipe mechanisms in the literature are differential and mechanical.

Poor hole cleaning and wellbore instability are major causes for mechanical sticking.Cutting transport is an essential part of the drilling operation. If the cuttings are not removed from the well properly, because of improper drilling fluid properties, they settle around the drill string causing the drill collars to become stuck. Also, hole cleaning is more important in directional wells due to formation of a cutting bed on the lower part of annulus. The directional well having an inclination angle between $30-60^{\circ}$ is the worst condition for hole cleaning [1].

Some causes for wellbore instability are as follows:

- Reactive shale formations

- Hole packing off

In the case of differential sticking, it has a direct relation to differential pressure between the hydrostatic column created by the drilling fluid in the well and formation pressure. Differential sticking occurs when high overbalanced drilling fluid exerts a large amount of differential pressure across a thick mud cake.
Usually, the first immediate action when the pipe suddenly gets stuck is to get it free as quickly as possible. The most common action in drilling to free the pipe is to work it out by firing the jars, no matter what type of stuck occurred. Particularly, in the case of differential stuck, it is very common practice to free the pipe by spotting fluids to be placed in the stuck point. These fluids can be water base, oil base or acid base. If all possible techniques to free stuck pipe are not helpful, backing off operation is performed. In order to back off pipe, estimation of free point of the stuck pipe is the key point. Two common approaches are utilized to locate the free point: pipe stretch calculations and free point (FP) logs that utilize magnetic properties of pipe or applying stretch and torque [2].

When the pipe is backed off and pulled out to surface, the fish or the remaining pipe in hole will be followed by fishing operations.

\subsection{Previous Studies}

Several approaches have been developed and utilized in the field of stuck pipe. Some of approaches are actually reactive techniques in which actions made after the pipe got stuck. Reactive methods include all attempts to free pipe after stuck occurred. Major parts of previous studies in stuck pipe belong to reactive methods. The other approaches are known as proactive or detective or also predictive. Predictive approaches try to detect and avoid stuck pipe to occur.

One of immediate actions done after pipe got stuck is firing jar, regardless of stuck mechanisms.Depending on causes of stuck pipe, jar can be applied downward or upward.

Gonzalez et al., 2007 [3] stated that jarring operations might last days or sometimes weeks, which maximizes the loss time. Resonant vibration can be alternative to jarring and some claim that it is more effective especially when it is installed and used directly after sticking occurs. This technique has advantages since it quickly frees the pipe and it is easy to apply. However, its effectiveness is minimized when the time of sticking exceeded 24 hours without applying this technique.

In the case of spotting and pumping pills, Halliday and Clapper in 1989 [4] specified the features of spotting fluid as to be 1) with low toxicity, 2) water miscibility, 3) adequate barite suspension, 4) no adverse effects, 5) moderate cost.

In 2008, Agarwal and Agarwal proposed [5] a new design of drill collar that would reduce the risk of differential sticking. This drill collar was designed in a way that could reduce the contact area with the filter cake, which in turn would reduce the force required to free the pipe.

The idea of using multivariate statistical analysis (MSA) to predict stuck pipe was first brought by Hempkins et al. through a research done in 1987 [6]. This research included 131 stuck pipe cases and 20 drilling variables. They utilized discriminant analysis to develop discriminant functions which 
are set of equations derived from correlations based on relationships between dependent and independent drilling parameters that would lead to stuck pipe.

In 2010, Shadizadeh et al. [7] conducted a research to investigate stuck pipe probability by ANN in one of Iranian oil fields. The results of their study showed more than $90 \%$ accuracy for stuck pipe prediction in the investigated oilfield. In their study, a total number of 275 cases were collected from the daily drilling reports (DDRs) in one of the Iranian oil fields. The data contained 115 stuck and 160 non-stuck cases. Non-stuck data were collected from days that the wells were completely safe and had not become stuck in the same general areas of operation. The stuck pipes were divided into dynamic and static types. In dynamic condition, the drilling fluid is in circulation, while it is not circulating during static condition.

There are two methods to determine Free Point, stretch method and free point indicator tool which are described below.

\section{1) Stretch Method}

Stuck point can be established by stretching the string and using the stretch charts for drill pipe. This method gives only an approximate value for the free point and should only be used to find the approximate stuck point for spotting pipe free pills and providing a starting point for using the Free Point Indicator Tool (FPIT).

$L=\frac{735294 \times \mathrm{W} \times \Delta \mathrm{L}}{\Delta \mathrm{F}}$

$\mathrm{L}=$ Length of Free Pipe (ft)

$\Delta \mathrm{L}=$ Length of Stretch (in)

$\Delta \mathrm{F}=$ Incremental Force or differential pull (lbs)

$\mathrm{W}=$ Weight of Drill Pipe (lb/ft)

\section{2) Free Point Indicator Tool (FPIT)}

This tool can be run on electrical wireline in order to backoff the string at the deepest possible point. By applying stretch and torque on the pipe, the FPIT can determine elongation or rotation at any depth. A plot of depth versus the percentage of surface torque and pull transmitted down hole shows the deepest point at which the string is free. Performed research in the case of free point estimation are in field of logging tools and modifying or utilizing new tools and techniques [8,9]. This study focuses on a novel topic of free point estimation, by using drilling parameters.

\section{ANN Free Point Prediction Model's Comparison to Conventional Methods}

It is claimed that ANN model for free point prediction would be a novel technique compared to conventional models in predicting free point. Conventional methods in estimating FP have some limitations which are listed below:

1) The traditional free-point (FP) logs are based on a measurement of strain when stress is applied to the pipe between two points. These measurements are taken at specific intervals and require the tool arms to firmly grip the pipe to prevent movement that could mask the strain measurement. This simple method is difficult to implement because it depends on the skill of the specialist operator.

2) The stretch-related amplitude measured by the tool downhole was determined by the tension applied at surface, the pipe friction, the material elastic properties, the thickness, and the size of the specific pipe in the bottom hole assembly (BHA). Some parameters including the friction and specific response of the particular completion piece, were so different that the service was performed by specialists that, based on experience, were able to understand the tool response under different conditions and the relation to the different stuckpipe mechanisms.

3) In new magnetic methods, presence of iron fillings or other metallic particles in the mud system tool response becomes highly active and erratic.

Noticing above limitations and considering high costs and operation time spent on wireline logging operation in stuck pipe condition, which time plays an important role, clarifies the importance of developing a new technique. ANN prediction of FP is less expensive and acceptable accuracy of the prediction made it a novel technique.

\subsection{ANN Description and Training}

Artificial neural network is an information-processing system that is a simulation of biological learning process [10]. A multilayer neural network consists of several layers. An input layer, in which input data are presented to the network, output layer which generates outputs of the network and hidden layer(s) between input and output layer. The basic elements of an ANN are neurons and their connection strengths (weights). The input to each neuron is multiplied by its associated weighting factor and then summed with the product of each other input nodes and their respective weighting factors. Activation functions are mainly used for the neurons in the hidden layer and output layer and constrain the neuron's output signal to fall within a fixed range $(0,1$ or $-1,1)$.

There are several types of ANNs; the most common types are the feed-forward back-propagation architectures which were used in this study. A feed-forward network has a layered structure and feed-forward topology. The term backpropagation refers to mechanism of adjusting weights for reduction of error. The error (the difference between predicted and actual outputs (d)) is computed and the goal of network is to reduce the error.

$E(x i, w i, d i)=\sum_{j=1}^{\infty}\left(\frac{1}{\mathrm{~N}_{j}}(\mathrm{y}(x i, w i)-d i)^{2}\right)$

$E$ is the error of network or Mean Square Error (MSE) for the output neurons $\left(\mathrm{N}_{\mathrm{j}}\right)$. When the network error is computed, the back propagation algorithm adjusts the weights (w) in the opposite direction of error gradient. The size of changes in weights is controlled by the parameter called the learning rate $(\eta)$. This method is known as gradient descent.

$\Delta w j i=-\eta \frac{\partial E}{\partial w j i}$

In a typical neural data-processing procedure, the database is divided into three separate portions called training, validation, and testing sets. The training set is used to develop the desired network. In this process, the desired output in the training set is used to help the network adjust the weights between its neurons or processing elements [10]. Network gradually learns and the error between predicted output and actual output decreases. Training the network too much would increase the risk of memorizing (overfitting). Validation data are used to avoid network memorizing the data so that training stops when error of validation data shows an increasing trend. An increase in error is a good indication of the point in which network has started to memorize patterns. Finally, the network must be capable of generalization. To generate reasonable 
output for new inputs, to ensure such network capability, a previously unseen testing data subset is presented to network. Seven important parameters affecting stuck pipe are considered in the ANN developed models. Also, its performance is compared with actual field data.

\section{MTERIAL AND METHODS}

So far, ANN has been widely used in petroleum engineering in the case of complex problems. If there is plenty of data and problem is poorly understood to derive a suitable model, the ANN technology is a good choice. In this study, ANN was used to predict FP to remove weak points and limitations of previous models discussed above. ANN was selected because it learns through actual input and output data and recognizes the real relation between parameters not based on assumptions and simplifications which are common in conventional modeling. Then, the model prediction performance is compared with field results. In this study, Tan-sigmoid activation function was usedand training data subset contained $70 \%$ of data base, validation data and testing data each one contained $15 \%$ of the remaining data.

\subsection{Data Base Acquisition and Selection of Parameters}

A total number of 76 cases were collected from the daily drilling reports (DDRs) in Iranian oil fields. The data contained 76 stuck cases which were collected from more than 40 wells.

The parameters that were collected as the important parameters in stuck pipe at first steps were as follows:

Mud properties which are Mud Weight (MW), Plastic Viscosity (PV), Yield Point (YP), 10-Second Gel Strength and 10-Minute Gel Strength (GL), Fluidloss (conventional API or High Pressure High Temperature (HPHT) API) and Solid content. Depths, bottom hole assembly size and length, drill pipe size, hole size, Rate Of Penetration (ROP), Annular velocity, pipe rotation in Revolutions Per Minute (RPM) and Mud cake thickness. Influence of the input parameters is considered as the key point for developing ANN models. Introducing more input parameters than required will result in a large network size and consequently decrease learning speed and efficiency [11]. Since the drilling parameters that are involved in stuck pipe are numerous, it is essential to find the variables that are closely related to stuck pipe. In ANN studies, the following criteria have been mentioned [12]

1) There must be a spread of values of the parameter in the databases. This allows the neural network to more easily approximate the function.

2) The variable must not be dependent on other input variables only. A parameter may be dependent on other input variables, but must also be dependent ona parameter that is not an input variable. In this way, the variable will provide information about the well that is not already provided by the other variables.

In this work, the above criteria were considered, and finally, some parameters were selected and some were removed. Removed parameters are WOB, MW, Depth, API Fluid loss, Mud cake thickness, BHA size, drill pipe size and hole size. Among these variables, WOB, Size of BHA, drill pipe and hole were removed considering the first criterion. It means there was not spread value for these parameters. Fluid loss also has a variety and there are three types of values for API fluid loss: a) conventional API fluid loss, b) HTHP fluid loss, and c) finally, No-control in some cases. Obviously, these types differ considerably and cannot be considered as a single parameter. Also, converting these types into a single new parameter is difficult and may be impossible [13]. Other parameters were removed by considering the last criterion. For example, Mud cake thickness is dependent on solid percent. Depth is also removed from parameters because free point as output illustrates the effect of depth on stuck pipe. Selected parameters and range of data used in this study are presented in Table 1 .

Table 1. Range of selected parameters

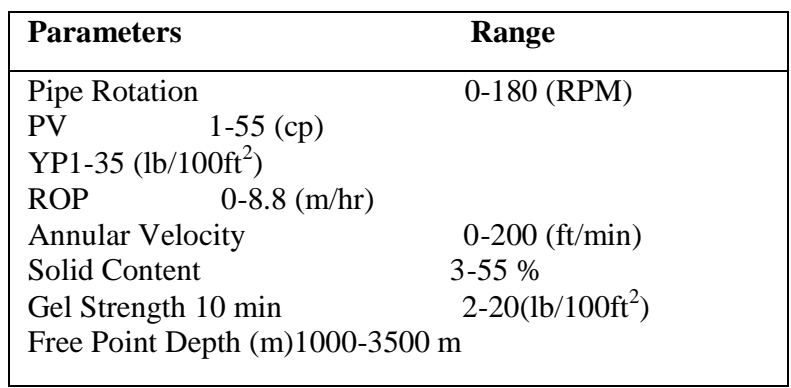

\subsection{Pre-processing}

Before applying the data base in neural network, it is necessary to do a pre-processing step. Normalizing data is one of the pre-processing routines in which increases network efficiency and ensures that all the parameters are in the same attention for the network. Applying the above procedure in this work resulted in significant improvement in the performance of the ANN. In this study, all input and output data are normalized between $[-1,1]$ by the function:

$y=\frac{\left(y_{\max }-y_{\min }\right)\left(x-x_{\min }\right)}{x_{\max }-x_{\min }}+y_{\min }$

Where $\mathrm{y}, \mathrm{y}_{\max }$ andy $\mathrm{m}_{\min }$ arenormalized values, 1 and -1 respectively.

\subsection{Network Architecture Design}

To develop ANN models, a three-layer feed-forward back propagation network has been used. Attempts were made to vary number of hidden layers and neurons of hidden layer. Increasing number of hidden layers had no effect on decreasing error but number of neurons affects error value and performance of network. The number of hidden neurons did not have any pre-determined constraints and so was determined by trial and error. Twenty neurons were found to be sufficient for the hidden layer. Therefore,number of neurons in hidden layer was set to 20 neurons. Illustration of neurons and design of network are shown in Table 2 and Table 3. In this study, training algorithm is LevenbergMarquardt (trainlm). This algorithm appears to be the fastest method for training moderate-sized feed-forward neural networks (up to several hundred weights).

Table 2. Best measurements of MSE vs. epoch

\begin{tabular}{|c|c|c|}
\hline Best Network & Training Subset & Validation Subset \\
\hline Epoch \# & 11 & 6 \\
\hline Minimum MSE & 0.002 & 0.04 \\
\hline
\end{tabular}

Table 3. Best performance vs. hidden layer neurons

\begin{tabular}{|c|c|c|c|c|}
\hline $\begin{array}{c}\text { No. of Hidden Layer's } \\
\text { Neurons }\end{array}$ & 8 & 20 & 35 & 40 \\
\hline $\begin{array}{c}\text { Best Performance(Min } \\
\text { MSE) }\end{array}$ & 0.14 & 0.0029 & 0.09 & 0.35 \\
\hline
\end{tabular}




\section{RESULTS AND DISCUSSION}

ANN developed model is comprehensive by considering most main effective parameters mentioned in literature and proved by field evidences, while some models do not consider some important parameters such as AV or Solid content.

The best performance (minimum error) for the training data subset and validation data subset are values of 0.002 and 0.04. And correlation coefficents for unseen data subset (testing data) is a value of 0.77 . the complete results for all data subsets are shown in Figures 1, 2 and 3 and Table 4. To report MSE, it is important to notice that normalized data are reversed and MSE is reported for unnormalized data. Because MSE of normalized data would be alower value and it is better to evaluate the network performance by reversed data.

Table 4.Correlation coefficient and mean square error of three data subsets.

\begin{tabular}{|c|c|c|c|}
\hline Result & Samples & MSE & R value \\
\hline Training data & 52 & 0.002 & 0.91 \\
\hline Validation data & 12 & 0.04 & 0.80 \\
\hline Testing data & 12 & 0.01 & 0.77 \\
\hline All data & 76 & - & 0.83 \\
\hline
\end{tabular}

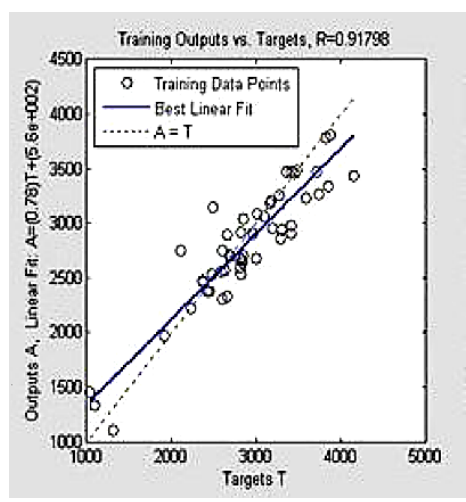

Figure 1: Linear correlation of training data subsets for free point prediction model.

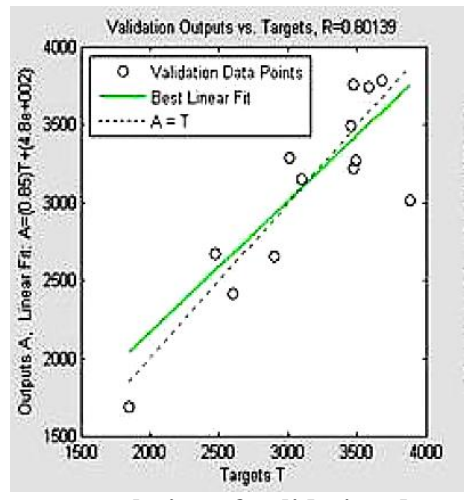

Figure 2: Linear correlation of validation data subsets for free point prediction model.

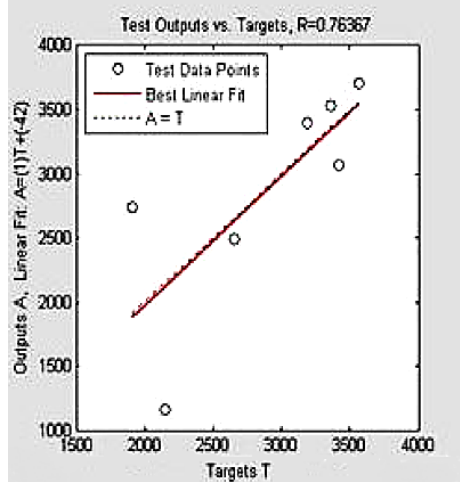

Figure 3: Linear correlation of testing data subsets for free point prediction model.

In Table 5, some important statistical properties are described, a comparison of errors, and correlation coefficent for three data subsets and all of data. As shown in Table 6, ANN results predict FP with less than $10 \%$ relative (percent) error compared to field results, and also there is no noticeable overprediction or under-prediction over entire range of data in ANN model. In some cases, error of prediction in these cases is not related to the networks performance. However, it is related to data or these kinds of sticking that are related to unpredictable sources. In one case, there is a noticeable error, it refers to the fact that the mean of data which network is trained by, is more than that value and most of FP data are above $2500 \mathrm{~m}$.

Table 5.Statistical properties of neural network performance.

\begin{tabular}{|c|c|c|c|}
\hline \multirow{2}{*}{ Data Sub Set } & \multicolumn{3}{|c|}{ Statistical Properties } \\
\cline { 2 - 4 } & APE & AAPE & R \\
\hline Training Data & -5.6 & 9 & 0.91 \\
\hline Validation Data & -8.05 & 14.5 & 0.80 \\
\hline Testing Data & -6 & 10.8 & 0.77 \\
\hline
\end{tabular}

Table 6. Percent error comparison of Ahwaz oil field's actual data to ANN predictions

\begin{tabular}{|c|c|c|}
\hline $\begin{array}{c}\text { Ahwaz } \\
\text { actual FP } \\
\text { data }\end{array}$ & $\begin{array}{c}\text { ANN } \\
\text { Predicted } \\
\text { data }\end{array}$ & $\begin{array}{c}\text { Percent } \\
\text { error \% }\end{array}$ \\
\hline 3336 & 3520 & 5.5 \\
\hline 3188 & 3370 & 5.7 \\
\hline 3566 & 3690 & 3.4 \\
\hline 3417 & 3171 & 7.2 \\
\hline 2655 & 2495 & 6 \\
\hline 3700 & 3542 & 4.2 \\
\hline 2147 & 1301 & 39 \\
\hline 3823 & 3442 & 10 \\
\hline 3085 & 3308 & 7.2 \\
\hline 3719 & 3600 & 3.2 \\
\hline 3583 & 3752 & 4.7 \\
\hline 3410 & 3220 & 5.5 \\
\hline
\end{tabular}




\section{CONCLUSIONS}

1) For the first time, ANN was utilized in stuck pipe field and a model developed for FP prediction. ANN model and conventional methods (all types ofFP logs and tools) can both predict FP but ANN has several preferences.

2) The value of correlation coefficients for the field and predicted data for FP model is 0.82. The obtained performances (MSE)are below 0.002. The statistical error analysis results obtained by the model indicate that ANN model is successful in predicting FP.

3) ANN developed model has an acceptable accuracy.

4) The main application of using these models is wireline logging time reduction during drilling operation.

\section{REFERENCES}

[1] Drilling Fluid Engineering Manual, MI L.L.C., Page: 15.1-15.10. March (1998).

[2] Torne, J., Rourke, M., Derouen, B. and Kessler, C., Middle East Case-Study Review of a New Free-Pipe Log for Stuck-Pipe Determination and Pipe-Recovery Techniques, SPE 147859, SPE Asia Pacific Oil and Gas Conference and Exhibition held in Jakarta, Indonesia, 20-22 September 2011.

[3] Gonzalez, O., Bernat, H. and Moore, P., The Extraction of Mud-Stuck Tubulars Using Vibratory Resonant Techniques, Paper SPE 109530 presented at the SPE Annual Technical Conference and Exhibition, Anaheim, California, U.S.A., 11-14 November 2007.

[4] Halliday, W. and Clapper, D., Toxicity and Performance Testing of Non-Oil Spotting Fluid for Differentially Stuck Pipe, Paper SPE/IADC 18684 presented at the SPE/IADC Drilling Conference, New Orleans, Louisiana, U.S.A., 28 February-3 March 1989.

[5] Agarwal, S.and Agarwal, N., Auto Release Drill Collars, Paper SPE 112348 presented at the Indian Oil and Gas Technical Conference and Exhibition, Mumbai, India, 46 March 2008.

[6] Hempkins, W. B., Kingsborough, R. H., Lohec, W. E. and Nini, C. J., Multivariate Statistical Analysis of Stuck
Drillpipe Situations, SPE 14181, SPE Drilling Engineering, September 1987

[7] Shadizadeh, S. R., Karimi, F. and Zoveidavianpoor, M., Drilling Stuck Pipe Prediction in Iranian Oil Fields: An Artificial Neural Network Approach, Iranian Journal of Chemical Engineering Vol. 7, No. 4 (Autumn), IAChE, 2010.

[8] Kessler, C., Dorffer, D., Crawford, D., Dehart, R. and Weiser, J., Case Histories of a New Wireline Logging Tool for Determination of Free Point in Support of Drilling and Pipe Recovery Operations. SPE/IADC 119907, presentation at the SPE/IADC Drilling Conference and Exhibition held in Amsterdam, The Netherlands, 17-19 March 2009.

[9] Mohanna, A., Voghell, M., Alali, A., Bakir, R. and AlMousa, A., A Step Change in Fishing Efficiency: Recovering Stuck Pipe Using the Fishing Agitation Tool, SPE 160880, presentation at the SPE Saudi Arabia Section Technical Symposium and Exhibition held in AlKhobar, Saudi Arabia, 8-11 April 2012.

[10] Mohaghegh, S. D., Virtual Intelligence Applications in Petroleum Engineering: Part 1-Artificial Neural Networks, paper SPE 58046, JPT, 64, (September 2000).

[11] Firoozi, M., Prediction of Bit Wear Using Artificial Neural Network, Ms Thesis, PUT, 2011.

[12] Goda, H. M., Maier, H.R., and Behrenbruch, P., "The development of an optimal artificial neural network model for estimating initial, irreducible water saturationAustralian reservoirs", Paper SPE 93307, Presented at the Asia Pacific Oil \& Gas Conference and Exhibition held in Jakarta, Indonesia, 5-7 April (2005).

[13] Farshad, F., Garber, J. D. and Lorde, J. N., "Predicting temperature profiles in producing oil wells using artificial neural networks", Paper SPE 53738, Presented at the Sixth Latin American and Caribbean Petroleum Engineering Conference held in Caracas, Venezuela, 2123 April, (1999). 